\title{
A systematic review of the psychometric properties, usability and clinical impacts of mobile mood-monitoring applications in young people
}

\author{
M. Dubad ${ }^{1 *}$, C. Winsper ${ }^{1}$, C. Meyer ${ }^{1,2}$, M. Livanou ${ }^{1}$ and S. Marwaha ${ }^{1,3}$ \\ ${ }^{1}$ Mental Health and Wellbeing, Division of Health Sciences, Warwick Medical School, University of Warwick, Coventry, UK \\ ${ }^{2}$ Warwick Manufacturing Group, University of Warwick, Coventry, UK \\ ${ }^{3}$ Affective Disorders Service, Caludon Centre, Coventry, UK
}

Background. Mobile mood-monitoring applications are increasingly used by mental health providers, widely advocated within research, and a potentially effective method to engage young people. However, little is known about their efficacy and usability in young populations.

\begin{abstract}
Method. A systematic review addressing three research questions focused on young people: (1) what are the psychometric properties of mobile mood-monitoring applications; (2) what is their usability; and (3) what are their positive and negative clinical impacts? Findings were synthesised narratively, study quality assessed and compared with evidence from adult studies.

Results. We reviewed 25 articles. Studies on the psychometric properties of mobile mood-monitoring applications were sparse, but indicate questionable to excellent internal consistency, moderate concurrent validity and good usability. Participation rates ranged from $30 \%$ to $99 \%$ across studies, and appeared to be affected by methodological factors (e.g. payments) and individual characteristics (e.g. IQ score). Mobile mood-monitoring applications are positively perceived by youth, may reduce depressive symptoms by increasing emotional awareness, and could aid in the detection of mental health and substance use problems. There was very limited evidence on potential negative impacts.

Conclusions. Evidence for the use of mood-monitoring applications in youth is promising but limited due to a lack of high-quality studies. Future work should explicate the effects of mobile mood-monitoring applications on effective selfregulation, clinical outcomes across disorders and young people's engagement with mental health services. Potential negative impacts in this population should also be investigated, as the adult literature suggests that application use could potentially increase negativity and depression symptoms.
\end{abstract}

Received 2 September 2016; Revised 19 May 2017; Accepted 23 May 2017; First published online 23 June 2017

Key words: Ecological momentary assessment, mental health, mhealth, mobile application, mood, mood monitoring, young people.

\section{Introduction}

Mood is an affective dynamic, which naturally varies across time and contexts (Trull et al. 2015). Problems with regulating mood can play a key role in the development and trajectory of a range of psychopathologies (Paris, 2004; Crowell et al. 2009; Marwaha et al. 2015). Traditionally, mood has been assessed with retrospective measures (Trull et al. 2015). This can increase the risk of recall bias subsequently reducing accuracy (Schwartz et al. 1999; Reid et al. 2009). The relatively

* Address for correspondence: M. Dubad, BSc, MRes, Mental Health and Wellbeing, Division of Health Sciences, Warwick Medical School, University of Warwick, Gibbet Hill Road, Coventry CV4 7AL, UK.

(Email: m.dubad@warwick.ac.uk) recent use of ecological momentary assessment (EMA) facilitates the real-time assessment of mood by collecting data on multiple occasions throughout the day (Wenze \& Miller, 2010). Thus, it may be more suitable for understanding daily mood changes (Cristobal-Narvaez et al. 2016; Myin-Germeys et al. 2016; van Knippenberg et al. 2016).

Various EMA techniques exist, ranging from paper-and-pencil to physiological assessment (Wenze \& Miller, 2010) to digital data collection. A number of UK governmental reports (HM Government, 2011; Department of Health, 2013) highlight the benefits of digital tools and Information and Communications Technology (ICT) in aiding the objective, reliable assessment and care of mental health problems. With demand for mental health services outgrowing available resources (Department of Health, 2013), technology might relieve some of this pressure by providing

This is an Open Access article, distributed under the terms of the Creative Commons Attribution licence (http://creativecommons.org/licenses/by/4.0/), which permits unrestricted re-use, distribution, and reproduction in any medium, provided the original work is properly cited. 
remote resources that increase access to effective treatment while reducing clinician load.

Applications ('apps') offer great promise to young people who are disproportionately affected by mental illness or may struggle to engage with mental health services (Seko et al. 2014). Apps are delivered in a medium young people are familiar with. Figures from Ofcom (2015) indicate that $90 \%$ of youth between the ages of 16 and 24 own a smartphone, regardless of sociodemographic domain. Given this widespread ownership and apparent attachment to mobile technology (Ofcom, 2015), youths might feel more comfortable with assessments and treatments utilising mobile apps.

Mental health services increasingly use apps (Olff, 2015), many of which have the capacity for EMA to monitor mood (e.g. Sandstrom et al. 2016b). Several reviews with mainly adult studies (e.g. Donker et al. 2013; Naslund et al. 2015; Nicholas et al. 2015; Torous \& Powell, 2015; Bakker et al. 2016; Faurholt-Jepsen et al. 2016; Walsh et al. 2016) have appraised evidence for the use of mood-monitoring apps.

Studies included in these reviews provide some evidence for the psychometric properties, e.g. internal consistency (Palmier-Claus et al. 2012) and concurrent validity (Faurholt-Jepsen et al. 2014) of these apps. There is also evidence for usability (Bardram et al. 2013). Participation rates are generally high across studies sampling adults, ranging from 65\% (Depp et al. 2015) to $88 \%$ (Ainsworth et al. 2013), though Depp et al. (2012) reported much higher completion rates for paper and pencil compared with app measures $(82.9 \%$ v. $42.1 \%)$. Evidence also suggests that apps may help people with mental health problems to monitor triggers (Bardram et al. 2013), that the capacity to convey experience can be therapeutic, and that apps could be a useful tool for improving patientclinician communication (Palmier-Claus et al. 2013).

Less is known about the use of mental health apps, particularly mood-monitoring apps, in youth (10-24 years). A scoping review by Seko et al. (2014) suggested that mood-monitoring apps are positively perceived by youth (Matthews et al. 2008a), may improve treatment adherence (Matthews et al. 2008b) and possibly improve mental wellbeing (Kauer et al. 2012). While intriguing, findings were preliminary due to the low quality of available evidence (NCCMH, 2014), the small number of studies on mood-monitoring apps specifically and the limited number of apps studied $(n=2)$ (NCCMH, 2014; Seko et al. 2014).

In summary, mood-monitoring apps offer a potentially important step change in the assessment of mood and delivery of youth mental health services. Despite this potential and the widespread advocacy for their use (e.g. Firth et al. 2016; Sandstrom et al. $2016 a$ ), there are no extant reviews examining the psychometric properties, usability and clinical impacts of mood-monitoring apps in young populations. Therefore, a systematic review was completed to address the following research questions: (1) what are the psychometric properties of mobile moodmonitoring apps; (2) what is their usability; (3) and what are their positive and negative clinical impacts among clinical and non-clinical youth populations? Our secondary aims were to frame our findings within the adult literature, and conduct a quality assessment to examine potential sources of bias.

\section{Method}

Following a scoping review, the authors developed the protocol delineating the planned methodology. The review was conducted in adherence to this protocol, and in line with the PRISMA statement (Moher et al. 2009).

\section{Information sources and search strategy}

The following sources were searched: Medline, EMBASE, PsycINFO, ProQuest Dissertations \& Theses, ProQuest SciTech Collection, the Association for Computing Machinery (ACM) Guide to Computing Literature and Web of Science for articles published from 2008 [the year when the first app was launched (Donker et al. 2013)]. Search terms were informed by previous reviews (Seko et al. 2014), and modified following advice from a medical librarian and field experts. The search was conducted by combining five groups of terms (see online Supplementary Table S1) relating to: type of technology (e.g. 'mhealth'), type of assessment (e.g. 'ambulatory assessment'), mood-related outcome or problem (e.g. 'bipolar disorder'), youth population (e.g. 'youth'), usability/treatment-related outcomes and psychometric properties (e.g. 'reliability', 'validity'). We were interested in all forms of validity potentially examined in the app literature, e.g. concurrent, face or predictive (Faurholt-Jepsen et al. 2016), though we anticipated a paucity of studies due to the novelty of the field. We defined the 'usability' of mood-monitoring apps in accordance with the International Organisation for Standardisation (2001) definition of usability, i.e. 'the capability of the software product to be understood, learned, used and attractive to the user, when used under specified conditions'. Consistent with previous systematic reviews (Donker et al. 2013), we included young people's participation rates (i.e. compliance, response and completion) and how apps were perceived by youths (including their acceptability - how satisfied they were with the app, whether it could be used with ease) as markers of usability. 
MD conducted a hand search of articles published in Cyberpsychology, Behavior and Social Network, the Journal of Medical Internet Research (JMIR), the JMIR Mental Health, and the JMIR mHealth and uHealth over the last 5 years. An additional search of the first 15 pages of Google Scholar was conducted (search terms 'mood', 'phone', 'app' and 'monitoring'). Reference lists and in-text citations of relevant articles were inspected. Finally, subject experts were approached to identify additional articles.

\section{Study selection}

Inclusion criteria were:

(1) Apps must have been developed for, and delivered through, mobile phones or smartphones;

(2) Participants aged 10-24 years (consistent with the World Health Organisation's definition of young people; World Health Organisation, 1986);

(3) Studies included published and unpublished research reported in the grey literature;

(4) Studies must have been published in the English language;

(5) Studies must have been published in 2008 or later;

(6) Studies must have included community or clinical populations (to ensure the inclusion of sub-clinical youth, who may subsequently access care).

\section{Screening procedure}

Following removal of duplicates, MD and ML independently screened $100 \%$ of titles and abstracts for fulltext retrieval. MD assessed full-text articles against the inclusion criteria and extracted relevant data.

\section{Quality assessment}

MD evaluated the quality of included studies for potential risk of bias using Cochrane's risk of bias tool, in which studies are allocated a rating of high, low or unclear risk of bias (Higgins et al. 2011).

\section{Data synthesis}

Quantitative and qualitative data were synthesised narratively.

\section{Results}

\section{Study selection}

A total of 1747 articles were identified in the initial search, and 19 from the hand search (Fig. 1). Following removal of duplicates, 1176 abstracts were screened, 86 of which were selected for full-text retrieval. There was a high level of agreement between raters $(\kappa=0.90)$. In total, 64 articles were excluded following full-text review. Three additional articles were identified following inspection of included studies. Twenty-five articles were included in the final review.

\section{Study characteristics}

Table 1 outlines study methodology, the characteristics and features assessed in the studies, and main findings. Three studies reported on a randomised controlled trial (RCT): one was the primary RCT (Reid et al. 2011), and two reported secondary analyses with the same dataset (Kauer et al. 2012; Reid et al. 2013). The remaining studies were non-experimental or quasi-experimental. The search identified 19 published studies and six unpublished studies (four conference proceedings; two theses). The majority of studies $(n=16)$ were quantitative; the remaining nine employed mixed methods.

Sample size ranged from 6 to 108996 participants. Eight studies recruited healthy participants. Eleven studies recruited participants from clinical populations including youth with a range of mental health, emotional or behavioural problems, such as depression $(n=8)$, high-functioning autism/Asperger's disorder $(n=2)$ and substance or alcohol use $(n=1)$. The remaining six studies recruited participants from mixed populations comprising healthy, mentally ill or substance-using individuals. Mean ages across studies ranged from 10.95 to 23.7 years.

Methods across studies varied greatly. For example, some studies lent participants a phone, whereas others let participants use their own device. Please see Table 1 for a description of the different data collection methods used in each study. As observed in the adult literature, terminology also varied greatly across studies (please see Usability section for more details).

Various apps were used, the most frequent of which was the 'Mobiletype' programme (Reid et al. 2009). Mood outcomes were either direct mood assessments, or described mood-related constructs or behaviours (e.g. stress, hostility). Outcomes were monitored over variable time periods. The shortest period was $24 \mathrm{~h}$ (Bossmann et al. 2013), the longest 326 days (Matthews \& Doherty, 2011). Monitoring schedules also varied, and could comprise hourly, daily or weekly monitoring, or requirements to complete measures a fixed number of times per day (with or without pre-specified time intervals). Reimbursements or incentives were available in 18 studies (e.g. payments, gift vouchers).

\section{Psychometric properties of mood-monitoring apps}

Nine studies reported on the reliability or validity of mood-monitoring apps. 


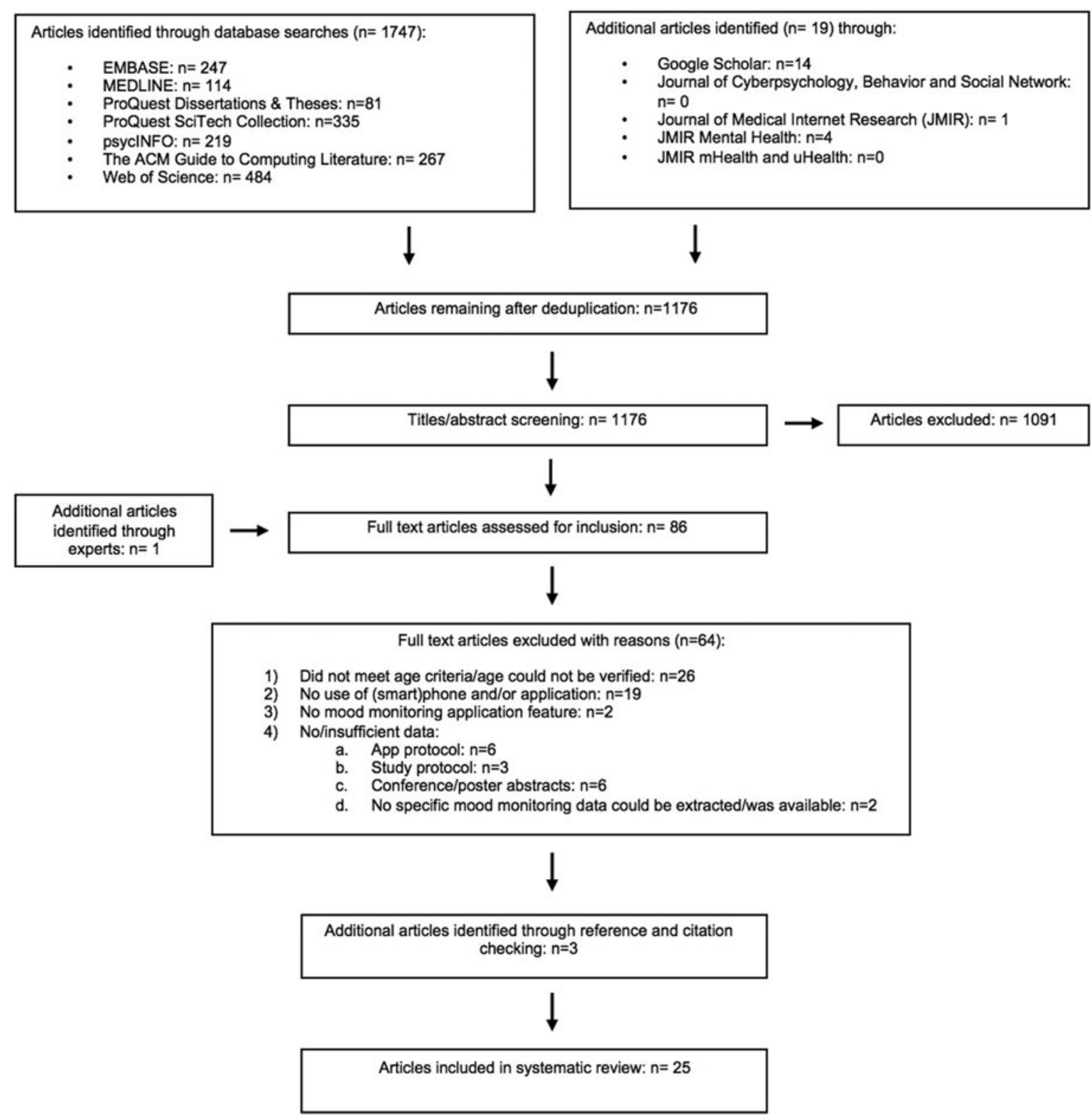

Fig. 1. Flowchart of literature search results and selection of studies.

\section{Reliability}

The internal consistency (correlation between items within a scale) was assessed in four studies (Dunton et al. 2011, 2014; Huh et al. 2014; Ansell et al. 2015). As demonstrated in Table 2, levels ranged from questionable to excellent (George \& Mallery, 2003).

\section{Validity}

Concurrent validity. Three studies examined concurrent validity (the correlation between an assessment and a previously validated assessment of the same construct). Concurrent validity was mostly moderate across studies (see Table 1). Khor et al. (2014a) compared relationships between participant and parent-reported data from the retrospective Responses to Stress Questionnaire (Connor-Smith et al. 2000) and mobile app data recording participants' responses to stress. In two studies of university students, Ben-Zeev et al. (2015) and Wang et al. (2014) compared momentary app and retrospective questionnaire data on perceived stress.

Face validity. Two studies described participants' views on the face validity of the 'Mobiletype' app (see Table 1 for numerical details). Reid et al. (2012), using a sample with various mental health problems, found that the app was relatively successful in capturing participants' feelings and current situation. Khor et al. (2014a), using 
Table 1. Study details including the author (year,) study purpose, sample characteristics, intervention details and a summary of the main findings

\begin{tabular}{|c|c|c|c|c|}
\hline Author (year) & Study purpose & Sample characteristics & Intervention $^{\mathrm{a}}$ & Main findings \\
\hline $\begin{array}{l}\text { Ansell et al. } \\
\text { (2015) }\end{array}$ & $\begin{array}{l}\text { To explore the effects of marijuana use on } \\
\text { impulsivity and hostility in everyday life } \\
\text { using smartphone-based EMA }\end{array}$ & $\begin{array}{l}\text { - Sample size: } N=43(M=23.7 \\
\text { years) } \\
\text { - Population type: young } \\
\text { recreational substance users } \\
\text { - Comparison/control: none } \\
\text { - Location: USA } \\
\text { - Data collection: in-person research }\end{array}$ & $\begin{array}{l}\text { - App name: not specified } \\
\text { - Operation system: not specified } \\
\text { - Accessibility: no web/general/app store } \\
\text { access } \\
\text { - Device: not specified } \\
\text { - Measurements: daily alcohol, tobacco and } \\
\text { marijuana use; daily impulsivity and daily } \\
\text { interpersonal hostility } \\
\text { - Monitoring period: } 14 \text { days, monitoring } \\
\text { schedule varied. Compliance monitored for } \\
\text { irregularities by research staff } \\
\text { - Incentive/reimbursement: payments + } \\
\text { bonus payment for } 95 \% \text { survey response } \\
\text { rate }\end{array}$ & $\begin{array}{l}\text { Psychometric properties: } \\
\text { - Reliability: acceptable to excellent internal } \\
\text { consistency } \\
\text { Usability: } \\
\text { - Participation rate: impulsivity: } 96 \% \text { completed } \\
\text { data; interpersonal interactions: }>99 \% \\
\text { completed data } \\
\text { Clinical impacts: } \\
\text { - Potential implications for problems with } \\
\text { (perceived) interpersonal hostility }\end{array}$ \\
\hline $\begin{array}{l}\text { Bachmann } \\
\text { et al. (2015) }\end{array}$ & $\begin{array}{l}\text { To examine the usability and } \\
\text { unobtrusiveness of the Mobile } \\
\text { Ambulatory Mood Assessment }\left(\mathrm{MoA}^{2}\right) \\
\text { app }\end{array}$ & $\begin{array}{l}\text { - Sample size: } N=9(M=23.4 \text { years }) \\
\text { - Population type: healthy/ } \\
\text { non-clinical participants } \\
\text { - Comparison/control: none } \\
\text { - Location: Germany } \\
\text { - Data collection: in-person research }\end{array}$ & $\begin{array}{l}\text { - App name: } \mathrm{MoA}^{2} \\
\text { - Operation system: Android } \\
\text { - Accessibility: no web/general/app store } \\
\text { access } \\
\text { - Device: participants used study phones } \\
\text { (Google Nexus 4) or personal Android } \\
\text { smartphone } \\
\text { - Measurements: mood, tiredness and stress } \\
\text { level } \\
\text { - Monitoring period: } 12 \text { prompts p/day for } 4 \\
\text { days } \\
\text { - Incentive/reimbursement: no payment }\end{array}$ & $\begin{array}{l}\text { Psychometric properties: not studied } \\
\text { Usability: } \\
\text { - Participants' perception: app perceived as } \\
\text { user-friendly and convenient } \\
\text { Clinical impacts: not studied/reported }\end{array}$ \\
\hline $\begin{array}{l}\text { Ben-Zeev } \\
\text { et al. }(2015)^{\text {c }}\end{array}$ & $\begin{array}{l}\text { To examine if smartphone sensor data can } \\
\text { be used to measure behaviour and mental } \\
\text { health }\end{array}$ & $\begin{array}{l}\text { - Sample size: } N=47(M=22.5 \\
\text { years) } \\
\text { - Population type: students } \\
\text { reporting varying levels of } \\
\text { depression symptoms } \\
\text { - Comparison/control: none } \\
\text { - Location: USA } \\
\text { - Data collection: in-person research }\end{array}$ & $\begin{array}{l}\text { - App name: StudentLife } \\
\text { - Operation system: Android } \\
\text { - Accessibility: no web/general/app store } \\
\text { access } \\
\text { - Device: participants were offered an } \\
\text { Android study smartphone - type not } \\
\text { specified } \\
\text { - Measurements: momentary stress and } \\
\text { automated sensor data } \\
\text { - Monitoring period: } 10 \text { weeks (sensor data } \\
\text { gathered automatically; stress ratings } \\
\text { completed daily, } 5 \text { days per week) } \\
\text { - Incentive/reimbursement: (Raffle) prizes }\end{array}$ & $\begin{array}{l}\text { Psychometric properties: } \\
\text { - Concurrent validity: significant moderate } \\
\text { relationship between averaged app-assessed } \\
\text { stress ratings and retrospective post-study } \\
\text { questionnaire scores on a measure of } \\
\text { perceived stress }(r=0.41, p<0.01) \\
\text { Usability: } \\
\text { - Participation rate: average weekly response } \\
\text { rate was } 4.92 \text { days a week }(98.4 \%) \\
\text { Clinical impacts: not studied/reported }\end{array}$ \\
\hline
\end{tabular}

Measurements: daily alcohol, tobacco and ulsivity and daily

irregularities by research staff

Incentive/reimbursement: payments

us payment for $95 \%$ survey response

App name: $\mathrm{MoA}^{2}$

Accessibility: no web/general/app store

access

(Google Nexus 4) or personal Android

Measurements: mood tiredness and stress

level days

centive/reimbursement: no payment

App name: StudentLife

- Accessibility: no web/general/app store access

Device: participants were offered an Android study smartphone - type not

automated sensor data

gathered automatically; stress ratings

- Incentive/reimbursement: (Raffle) prizes
Psychometric properties:

relationship between averaged app-assessed

stress ratings and retrospective post-study

questionnaire scores on a measure of

sability:

- Participation rate: average weekly response rate was 4.92 days a week $(98.4 \%)$ 


\begin{tabular}{|c|c|c|}
\hline $\begin{array}{l}\text { Bossmann } \\
\text { et al. (2013) }\end{array}$ & $\begin{array}{l}\text { To clarify the relationship between } \\
\text { everyday physical activity and affective } \\
\text { states over a 1-day period }\end{array}$ & $\begin{array}{l}\text { - Sample size: } N=62(M=21.4 \\
\text { years) } \\
\text { - Population type: healthy/ } \\
\text { non-clinical students } \\
\text { - Comparison/control: none } \\
\text { - Location: Germany } \\
\text { - Data collection: in-person research }\end{array}$ \\
\hline $\begin{array}{l}\text { Crooke et al. } \\
(2013)\end{array}$ & $\begin{array}{l}\text { To examine the relationship between } \\
\text { varying rates of alcohol use and positive } \\
\text { and negative mood through EMA }\end{array}$ & $\begin{array}{l}\text { - Sample size: } N=41 \text { ( } M=15.4 \\
\text { years) } \\
\text { - Population type: young people } \\
\text { with varying levels of alcohol } \\
\text { intake } \\
\text { - Comparison/control: none } \\
\text { - Location: Australia } \\
\text { - Data collection: } \text { in-person researo }\end{array}$ \\
\hline
\end{tabular}

Dennis et al. To assess the feasibility of

(2015) smartphone-based EMA and recovery support ecological momentary interventions (EMI) via smartphones. The study also assessed the feasibility of using EMA and EMI to predict substance use in the following week

Location: Australia

- Data collection: in-person research

- Sample size: $N=29(M=16.6$ years)

- Population type: adolescents with different clinical problems

- Comparison/control: none

- Location: USA

- Data collection: in-person research
- App name: MyExperience movisens Edition version 594

- Operation system: Android

- Accessibility: web/general access only

- Device: participants were provided with an HTC Touch 2 smartphone

- Measurements: valence, calmness and energetic arousal

- Monitoring period: 1 day - affect

measurements every hour after waking up

- Incentive/reimbursement: no payment

- App name: Mobiletype

- Operation system: not specified

- Accessibility: no web/general/app store

access

- Device: participants were lent a Nokia 6630

- Measurements: activities, company,

location, mood, responses to stressful events and coping, and questions on participants' previous evening's alcohol and cannabis use

- Monitoring period: $4 \times \mathrm{p} /$ day on 20 randomised days over the 31-day study period

- Incentive/reimbursement: Partial reimbursement/ gift voucher (value: \$25)

- App name: Addiction Comprehensive Health Enhancement Support System (ACHESS)

- Operation system: Android

- Accessibility: web/general access only

- Device: participants provided with a smartphone - type not specified

- Measurements: feelings, activities, location and social context, and drug and alcohol related measurements

- Monitoring period: $6 \times$ p/day for 6 weeks. Compliance monitored for irregularities by research staff

- Incentive/reimbursement: payment -up to $\$ 50$ per week for adherence to all study requirements

- App name: MyExperience

- Operation system: Windows

- Accessibility: web/general access only

- Device: participants were lent an HTC Shadow.
Psychometric properties: not studied

Usability:

Participation rate:

Mean completion rate was 10.5 electronic diaries per participant

O Please note that 15 participants were excluded for missing data

Clinical impacts: not studied/reported

Psychometric properties: not studied

Usability:

- Participation rate: $58.3 \%$ (AM diaries) and $43.8 \%$ (PM diaries) completed mood assessments

Clinical impacts:

- Potential implications for youth alcohol interventions

Psychometric properties: not studied

Usability:

- Participation rate: $89 \%$ of assessments completed

- Participants' perception

O App-based EMA perceived as 'not too long' (95\%), 'very easy' or 'easy to learn how to do' (100\%), and 'very easy' or 'easy to complete six EMAs per day' (94\%)

$\bigcirc$ Of note, one participant withdrew early from the study due to frustrations with software problems

Clinical impacts:

- Potential implications for relapse prevention

Psychometric properties:

- Reliability: acceptable to good internal consistency $^{\mathrm{b}}$ varying body mass index (BM) levels 


\begin{tabular}{|c|c|c|c|c|}
\hline Author (year) & Study purpose & Sample characteristics & Intervention $^{a}$ & Main findings \\
\hline & & $\begin{array}{l}\text { - Comparison/control: none } \\
\text { - Location: USA } \\
\text { - Data collection: in-person research }\end{array}$ & $\begin{array}{l}\text { Measurements: main activity type, social } \\
\text { context, physical location, mood and } \\
\text { enjoyment } \\
\text { - Monitoring period: monitoring period: } 3-7 \\
\text { random prompts p/day within pre-specified } \\
\text { times over two data collection waves } \\
\text { (duration: } 4 \text { days per wave), separated by } \\
6 \text { months } \\
\text { - Incentive/reimbursement: up to } \$ 40 \\
\text { (compensatory) payment }\end{array}$ & $\begin{array}{l}\text { Usability: } \\
\text { - Participation rate: } 76 \% \text { of assessments } \\
\text { completed on average } \\
\text { Clinical impacts: not studied/reported }\end{array}$ \\
\hline $\begin{array}{l}\text { Dunton et al. } \\
(2011)^{\mathrm{d}}\end{array}$ & $\begin{array}{l}\text { To assess if the level and experience of } \\
\text { children's leisure-time physical activity } \\
\text { vary with social and physical contexts by } \\
\text { means of EMA }\end{array}$ & $\begin{array}{l}\text { - Sample size: } N=121(M=11.02 \\
\text { years) } \\
\text { - Population type: children with } \\
\text { varying BMI levels } \\
\text { - Comparison/control: none } \\
\text { - Location: USA } \\
\text { - Data collection: in-person research }\end{array}$ & $\begin{array}{l}\text { - App name: MyExperience } \\
\text { - Operation system: Windows } \\
\text { - Accessibility: web/general access only } \\
\text { - Device: participants were lent an HTC } \\
\text { Shadow. } \\
\text { - Measurements: main activity type, social } \\
\text { context, physical location, mood and } \\
\text { enjoyment } \\
\text { - Monitoring period: } 3-7 \text { random prompts p/ } \\
\text { day within pre-specified times over } 4 \text { days } \\
\text { - Incentive/reimbursement: up to } \$ 40 \\
\text { (compensatory) payment }\end{array}$ & $\begin{array}{l}\text { Psychometric properties: } \\
\text { - Reliability: acceptable to good internal } \\
\text { consistency } \\
\text { Usability: } \\
\text { - Participation rate: } 80.3 \% \text { of assessments } \\
\text { completed on average } \\
\text { Clinical impacts: not studied/reported }\end{array}$ \\
\hline $\begin{array}{l}\text { Huh et al. } \\
\text { (2014) }\end{array}$ & $\begin{array}{l}\text { To examine the contextual antecedents to } \\
\text { smoking in a sample of Korean American } \\
\text { young adult smokers through EMA }\end{array}$ & $\begin{array}{l}\text { - Sample size: } N=22(M=21.23 \\
\text { years }) \\
\text { - Population type: young adult } \\
\text { smokers } \\
\text { - Comparison/control: none } \\
\text { - Location: USA } \\
\text { - Data collection: in-person research }\end{array}$ & $\begin{array}{l}\text { - App name: ActiPal (MEI Ltd.) } \\
\text { - Operation system: Android } \\
\text { - Accessibility: web/general access only } \\
\text { (demo app) } \\
\text { - Measurements: affect, perceived stress, } \\
\text { cigarette craving, and other contextual and } \\
\text { environmental measures } \\
\text { - Device: Android enabled phones (study } \\
\text { phones provided if participants owned } \\
\text { iPhones) } \\
\text { - Monitoring period: random non-smoking } \\
\text { signal contingent (5× p/day for } 7 \text { days)+ } \\
\text { event-contingent prompts over a } 7 \text { day } \\
\text { period. Compliance closely monitored by } \\
\text { research staff } \\
\text { - Incentive/reimbursement: not reported }\end{array}$ & $\begin{array}{l}\text { Psychometric properties: } \\
\text { - Reliability: questionable to acceptable internal } \\
\text { consistency }{ }^{\mathrm{b}} \\
\text { Usability: } \\
\text { - Participation rate: } 92.4 \% \text { of assessments } \\
\text { completed on average } \\
\text { - Participants' perception: it should be noted } \\
\text { that one participant withdrew from the } \\
\text { study due to technical difficulties with the } \\
\text { EMA app } \\
\text { Clinical impacts: not studied/reported }\end{array}$ \\
\hline
\end{tabular}




\begin{tabular}{|c|c|c|c|c|}
\hline $\begin{array}{l}\text { Kauer et al. } \\
(2012)^{\mathrm{e}}\end{array}$ & $\begin{array}{l}\text { A secondary analysis that investigated the } \\
\text { relationships between self-monitoring, } \\
\text { emotional self-awareness, and depression } \\
\text { through EMA }\end{array}$ & $\begin{array}{l}\text { - Sample size: } N=69(M=18.5 \\
\text { years) } \\
\text { - Population type: young people } \\
\text { with mild or more severe mental } \\
\text { health/emotional problems } \\
\text { - Comparison/control: attention } \\
\text { comparison ( } n=49, M=17.4 \text { years). } \\
\text { - Location: Australia } \\
\text { - Data collection: In-person research }\end{array}$ & See Reid et al. (2011) & $\begin{array}{l}\text { Psychometric properties: not studied } \\
\text { Usability: } \\
\text { - Participation rate: completion rates were } \\
52.9 \% \text { for the intervention group and } 59.6 \% \\
\text { for the comparison group } \\
\text { Clinical impacts: implications for depression } \\
\text { symptoms }\end{array}$ \\
\hline $\begin{array}{l}\text { Kauer et al. } \\
(2009)^{\mathrm{f}}\end{array}$ & $\begin{array}{l}\text { To assess the feasibility and usefulness of a } \\
\text { mobile phone-based EMA app to gather } \\
\text { information on alcohol use and related } \\
\text { behaviours }\end{array}$ & $\begin{array}{l}\text { - Sample size: } N=18 \text { [mean ages } \\
15.9 \text { years (females) and } 15.8 \text { years } \\
\text { (males)] in study } 1 ; n=6 \text { [mean } \\
\text { ages } 18.3 \text { years (females) and } 19.5 \\
\text { years (males)] in study } 2 \\
\text { - Population type: healthy/ } \\
\text { non-clinical students in study } 1 \\
\text { and high-risk drinkers in study } 2 \\
\text { - Comparison/control: none } \\
\text { - Location: Australia } \\
\text { - Data collection: in-person research }\end{array}$ & $\begin{array}{l}\text { - App name: Mobiletype } \\
\text { - Operation system: not specified } \\
\text { - Accessibility: no web/general/app store } \\
\text { access } \\
\text { - Device: participants were lent a Nokia } 6630 \\
\text { - Measurements: activity, mood, stress, } \\
\text { alcohol and cannabis use } \\
\text { - Monitoring period: } 4 \times \text { p/day for } 1 \text { week } \\
\text { - Incentive/reimbursement: partial } \\
\text { reimbursement/gift voucher (value: } \$ 25)\end{array}$ & $\begin{array}{l}\text { Psychometric properties: not studied } \\
\text { Usability: } \\
\text { - Participation rate: better compliance for } \\
\text { school-based adolescents than older } \\
\text { adolescent high-risk drinkers } \\
\text { Clinical impacts: not studied /reported }\end{array}$ \\
\hline $\begin{array}{l}\text { Kenny et al. } \\
(2015)\end{array}$ & $\begin{array}{l}\text { To assess the feasibility of the CopeSmart } \\
\text { app }\end{array}$ & $\begin{array}{l}\text { - Sample size: } N=43(M=16.0 \\
\text { years) } \\
\text { - Population type: healthy/ } \\
\text { non-clinical adolescents } \\
\text { - Comparison/control: none } \\
\text { - Location: Ireland } \\
\text { - Data collection: in-person research }\end{array}$ & $\begin{array}{l}\text { - App name: CopeSmart } \\
\text { - Operation system: Android + iOS } \\
\text { - Accessibility: no web/general/app store } \\
\text { access } \\
\text { - Device: app was downloaded on } \\
\text { participants' Android or iOS phones } \\
\text { - Measurements: happiness, anger, sadness, } \\
\text { stress and worries } \\
\text { - Monitoring period: } 1 \text { week } \\
\text { - Incentive/reimbursement: no monetary } \\
\text { incentive }\end{array}$ & $\begin{array}{l}\text { Psychometric properties: not studied } \\
\text { Usability: } \\
\text { - Participation rate: participants engaged with } \\
\text { the app on } 4 \text { out of } 7 \text { days ( } 57.1 \%) \\
\text { - Participants' perception: the app's interface } \\
\text { layout was liked by } 79 \% \text { of participants. } \\
\text { Furthermore, the app was perceived as easy } \\
\text { to use ( } 93 \% \text {; minor technical difficulties with } \\
\text { logging on were experienced by } 7 \% \text { of } \\
\text { participants; } 70 \% \text { of participants would use } \\
\text { the app in the future; } 74 \% \text { believed the app } \\
\text { would be used by other young people; and } \\
70 \% \text { would recommend the app to a friend } \\
\text { Clinical impacts: } \\
\text { - Implications for self-awareness }\end{array}$ \\
\hline $\begin{array}{l}\text { Khor } \text { et al. } \\
(2014 a)^{\mathrm{g}}\end{array}$ & $\begin{array}{l}\text { To assess the utility of the Mobiletype } \\
\text { programme to examine adolescents with } \\
\text { High-Functioning Autism/Asperger's } \\
\text { Disorder's (HFASD) stressors and coping }\end{array}$ & $\begin{array}{l}\text { - Sample size: } N=31(M=14.46 \\
\text { years)+ parents } \\
\text { - Population type: adolescents with } \\
\text { HFASD } \\
\text { - Comparison/control: none } \\
\text { - Location: Australia } \\
\text { - Data collection: in-person research }\end{array}$ & $\begin{array}{l}\text { - App name: Mobiletype (adapted) } \\
\text { - Operation system: not specified } \\
\text { - Accessibility: no web/general/app store } \\
\text { access } \\
\text { - Device: participants were lent a Sony } \\
\text { Ericson } 7501 \mathrm{i} \\
\text { - Measurements: mood, stress, last time and } \\
\text { daily stress } \\
\text { - Monitoring period: } 4 \times \text { p/day for } 2 \text { weeks }\end{array}$ & $\begin{array}{l}\text { Psychometric properties: } \\
\text { - Concurrent validity: } \\
\text { O Mostly poor to moderate correlations } \\
\text { between data from the retrospective } \\
\text { Responses to Stress Questionnaire } \\
\text { (Connor-Smith et al. 2000) and mobile app } \\
\text { data recording participants' responses to } \\
\text { stress }\end{array}$ \\
\hline
\end{tabular}

Pychometric properties: not studied

group and $59.6 \%$

or the comparison group

Clinical impacts: implications for depression

. sability:

adolescent high-risk drinkers

- Device: participants were lent a Nokia 6630

Measurements: activity, mood, stress

Comparison/control: none

Data collection: in-person research

$N=43(M=16.0$

- App name: CopeSmart

Accessibility: no web/general/app stor

access

Measurements: happiness, anger, sadness,

- Incentive/reimbursement: no monetary

incentive

- Monitoring period: $4 \times \mathrm{p} /$ day for 2 weeks 
Intervention $^{\mathrm{a}}$

Main findings

Incentive/reimbursement: partial

reimbursement (value: \$20)
- Sample size: $N=6$ (ages 17-24, no - App name: Daybuilder

- Operation system: Android

- Accessibility: no web/general/app store access

- Device: participants provided with Android device with app installed
- Comparison/control: none

- Location: Denmark
O A significant moderate to strong correlation for the 'involuntary engagement' factor: $r=0.70, p<0.01$; parent report: $r=0.48, p<0.01$

$\bigcirc$ A significant strong correlation for the primary control engagement coping factor: $r=0.53, p<0.05$

- Face validity:

$\bigcirc$ The face validity was measured by assessing how well the app captured participants' current situation, thoughts and feelings

$\bigcirc$ The highest ratings were reported for the app's ability to capture participants feelings (67\%); followed by its ability to capture participants' current situation $(63 \%)$; and finally its ability to measure participants' thoughts $(50 \%)$

\section{Usability:}

- Participation rate: participants responded to $61.8 \%$ of prompts

O Note that a substantial proportion of participants gradually stopped responding throughout the study; while every participant completed at least one entry on the first day, completion rates reduced to $45 \%$ on day 14

O Also note that there was a significant positive correlation between full scale IQ and compliance rates $(r=0.46, p<0.01)$

Clinical impacts: not studied/reported

Psychometric properties: not studied

Usability: not studied/reported

Clinical impacts:

- Implications for emotional and behavioural problems

Psychometric properties: not studied

Usability:

- Participation rate: different compliance rates across app features -no obvious pattern.

Mean normalised compliance for daily registrations of approximately $30 \%$; mean 
- Data collection: in-person research - Measurements: Weekly Major Depression

Matthews \& To assess the issues around the use of

Doherty mobile phones for mood charting with the

(2011)

aim to improve adolescent engagement

To explore the effectiveness of mobile

Matthews et al. $(2008 b)$ phone $v$. pen-and-paper for mood tracking
- Sample size: $N=9(M=13.78$

- Population type: young people with depression, mood disorders, self-harm and anger management

- Comparison/control: non

- Location: Ireland

- Data collection: in-person research

- Sample size: $N=73(M=14.87$

years

- Population type: healthy/ non-clinical students

- Comparison/control: paper-based diary condition $(n=52)$

- Location: Ireland

- Data collection: in-person research

A study aimed at developing, piloting and reviewing a youth focused mobile phone programme to track young people's experiences in real time study [males ( $n=5, M=15.8$ years)
- Sample size: focus group ( $n=11$ mean age not reported) and pilot and females $(n=13, M=15$.

years)]

- Population type: students

- Comparison/control: none

- Location: Australia

- Data collection: in-person research Inventory; daily mood, appetite and sleep - Monitoring period: 4 weeks

- Incentive/reimbursement: payment of 500 DKK ( $\$ 95$ or $2 \mathrm{~h}$ salary)

normalised compliance for weekly registrations of approximately 50\%

- Participants' perception: user experience negatively affected by technological difficulties; clinicians highlighted the usefulness of self-monitoring when combined with therapy.

Clinical impacts:

- Implications for treatment

- App name: Mobile Mood Diary (MMD)

- Operation system: not specified

- Accessibility: no web/general/app store

access

Psychometric properties: not studied

Usability:

- Participation rate: $65 \%$ response on average

Clinical impacts:

- Device: app downloaded on clients' phones - Implications for treatment

- Measurements: energy, sleep and mood + free area for thought entries

Monitoring period: min. $1 \times$ p/day for two sessions

- Incentive/reimbursement: reimbursement where necessary

- App name: MMD

- Operation system: not specified

- Accessibility: no web/general/app store access

- Device: app downloaded on students phones

- Measurements: energy, sleep and mood + free area for thought entries

- Monitoring period: $1 \times$ p/day for 2 weeks

- Incentive/reimbursement: none

See Kauer et al. (2009)

(n)

(1)

Psychometric properties: not studied

Usability:

- Participation rate: mobile group significantly more responsive than paper-diary group $(t=-2.324, p<0.05)$

- Participants' perception: participants preferred mobile technology

Clinical impacts: not studied/reported

Psychometric properties: not studied

Usability:

- Participation rate:

O Participants' completed $76 \%$ of diaries

O However, response rates decreased from $91 \%$ on day 1 to $67 \%$ on day 7

$\bigcirc$ Of note, one-third of the sample stated that they did not always respond honestly to items if a specific response would result in further questioning

- Participants' perception: the study's initial response rate suggested mobile technology may not be preferred or adopted by all young people. Nevertheless, the app was 


\section{A randomised controlled trial to investigate some of the mental health benefits of the Mobiletype programme}

- Sample size: $N=68(M=18.5$ years)

- Population type: mild/more mental health or emotional problems

- Comparison/control: compariso programme ( $n=46, M=17.4$ years)

- Location: Australia

- Data collection: in-person research

\section{Reid et al. \\ $(2013)^{\mathrm{e}}$}

To assess the utility of Mobiletype in a

See Reid et al. (2011) primary care setting (secondary analysis)
- Sample size: $n=47(M=15.59$ years)

- Mental health/clinical status: adolescents with varied (medical) disorders.

- Comparison/control: none

- Location: Australia

- Data collection: in-person research
- App name: Mobiletype

- Operation system: not specified

- Accessibility: no web/general/app store access

- Device: participants were lent a Sony Ericsson Z750i mobile phone

- Measurements: current activities, company, location, mood, recent stressful events, responses to stressful events, alcohol consumption, cannabis use, and sleep, exercise and diet-related questions

- Monitoring period: $\mathrm{min} .2 \times /$ day for $2-4$ weeks

- Incentive/reimbursement: partial reimbursement $(\mathrm{A} \$ 30)$ and gift cards $(\mathrm{A} \$ 20)$ for post-questionnaires completion (maximum A $\$ 60$ ) See Reid et al. (2011)

\section{- App name: Mobiletype}

- Operation system: not specified

- Accessibility: no web/general/app store access

- Device: participants were lent a ZTE F851 JAVA MIDP 2.0 phone with $\$ 50$ credit

- Measurements: location, activity, company, mood, stressful events, responses to stressful events, alcohol and cannabis use, sleep, exercise and diet-related questions

- Monitoring period: four random prompts $\mathrm{p} /$ day for 2-4 weeks (min. completion: $1 \times \mathrm{p} /$ day) overall viewed as youth-friendly and non-invasive

Clinical impacts: not studied/reported

Psychometric properties: not studied Usability:

- Participation rate: response rates for the intervention group: $52.9 \%$; comparison group: $60.9 \%$

Clinical impacts:

- No significant effects on mental health outcomes; potential implications for self-awareness

Psychometric properties: not studied Usability: not studied/reported

Clinical impacts:

- Potential implications for treatment and clinicians' understanding of patients

Psychometric properties:

- Face validity:

O The face validity was measured by assessing how well the app captured participants' current situation, thoughts and feelings

The highest ratings were reported for the app's ability to capture participants' feelings ( $86 \%$ ); followed by its ability to capture participants' current situation $(83 \%)$; and finally its ability to measure participants' thoughts $(57 \%)$ 
- Incentive/reimbursement: none

Sacco (2015) To examine the feasibility and utility of a areas of functioning associated with depression UDTracker App

Operation system: Android smartphone app developed to assess five

- App name: Android Health and Wellness

- Accessibility: no web/general/app store access

- Device: app installed on participants' own Android enabled phones

- Measurements: depression, mood, social functioning, cognitive and lifestyle factors, coping/emotion regulation (daily or weekly)

- Monitoring period: 14 days. Assessment times varied across measures: $1 \times$ p/evening [e.g. Positive and Negative Affect Scale (Watson et al. 1988)], 1× p/morning [sleep questionnaire, adapted from Pittsburgh Sleep Quality Index (Buysse et al. 1989)], and $1 \times \mathrm{p}$ /week [e.g. items from the COPE scale (Carver et al. 1989)]

- Incentive/reimbursement: extra/research participation course credit

Scotti (2015) To assess the efficacy, acceptability and feasibility of the school-based Dialectical Behaviour Therapy skills group for the treatment of adolescent eating disorders and sub-diagnostic problematic eating behaviours

To describe a smartphone app for the self-monitoring of eating disorder symptoms, evaluate characteristics of app

Population type: students with eating disorder symptoms or body mage concerns

- Comparison/control: two high school students who had withdrawn ( $M=16.5$ years)

- Location: USA

- Sample size: $N=108996[M=22$ years (reported by 48830 users)]
App name: not specified

- Accessibility: unknown web/genera access, no app store access

Device: participants own smartphones type not specified

- Measurements: individual eating disorder-related behaviours and cognitions/ feelings

- Study/monitoring period: 12 weeks

- Incentive/reimbursement: academic credit

- App name: Recovery Record

- Operation system: Android + $\mathrm{iO}$
- Sample size: $N=114(M=19.36$ years)

- Population type: students with varying levels of depression symptoms

- Comparison/control: none

- Location: USA

- Data collection: in-person research
Usability:

- Participation rate: Participants completed $91 \%$ of entries in week 1

Clinical impacts:

- Potential implications for assessment and management

Psychometric properties: not studied

Usability:

- Participation rate: $85-93 \%$ response rate across different measures

- Participants' perception:

App perceived as 'easy to use' (95.6\%); 'a little' to 'not at all' irritating (90.3\%)

O The monotony of responding to the same survey questions (15\%); the high frequency of the pop-up notifications $(9 \%)$, and the drain on the phone's battery life $(8 \%)$ were perceived as irritating. Participants suggested more varied survey questions (23\%), fewer crashes, bugs or freezes $(9 \%)$ and provided

suggestions for novel technical features $(13 \%)$

O Some participants also enjoyed the user-friendliness of the app $(40 \%)$ and the pop-up-reminder feature $(17 \%)$

\section{Clinical impacts:}

- Potential implications for self-reflection on emotions or behaviours

Psychometric properties: not studied

Usability:

- Participants' perception: preference for paper-and-pencil tracking by some participants

Clinical impacts: not studied/reported

- Data collection: in-person research and/or prize draw 
Table 1 (cont.)

\begin{tabular}{|c|c|c|c|c|}
\hline Author (year) & Study purpose & Sample characteristics & Intervention ${ }^{a}$ & Main findings \\
\hline & $\begin{array}{l}\text { users and assess the feasibility and } \\
\text { utilisation of the app for self-monitoring } \\
\text { purposes }\end{array}$ & $\begin{array}{l}\text { - Population type: people with } \\
\text { varying levels of ED severity } \\
\text { - Comparison/control: none } \\
\text { - Study Location: USA } \\
\text { - Data collection: crowd-sourcing }\end{array}$ & $\begin{array}{l}\text { - Accessibility: general/web and app store } \\
\text { access } \\
\text { - Device: own (iOS or Android) smartphone } \\
\text { - type not specified } \\
\text { - Measurements: meals and eating } \\
\text { disorder-related behaviours/cognitions/ } \\
\text { feelings/urges } \\
\text { - Monitoring period: overall usage data not } \\
\text { available - six monitoring prompts p/day } \\
\text { - Incentive: none }\end{array}$ & $\begin{array}{l}\text { Usability: } \\
\text { - Participation rate: } 89 \% \text { of participants } \\
\text { monitored } \geqslant 3 \text { meals; } 67 \% \text { continued to } \\
\text { monitor at } 30 \text { days } \\
\text { - Participants' response: app received high user } \\
\text { ratings } \\
\text { Clinical impacts: not studied/reported }\end{array}$ \\
\hline $\begin{array}{l}\text { Wang et al. } \\
(2014)^{\mathrm{c}}\end{array}$ & $\begin{array}{l}\text { To measure university students' mental } \\
\text { health, academic performance and } \\
\text { behavioural trends using the StudentLife } \\
\text { app }\end{array}$ & $\begin{array}{l}\text { - Sample size: } N=48(M=22.8 \\
\text { years) } \\
\text { - Population type: university } \\
\text { students with varying depression } \\
\text { scores. } \\
\text { - Comparison/control: none } \\
\text { - Location: USA } \\
\text { - Data collection: in-person research }\end{array}$ & $\begin{array}{l}\text { - App name: StudentLife } \\
\text { - Operation system: Android } \\
\text { - Accessibility: no web/general/app store } \\
\text { access } \\
\text { - Device: participants either used their own } \\
\text { Android phones (primary users) or were } \\
\text { offered an Android Nexus 4a (secondary } \\
\text { users) } \\
\text { - Measurements: momentary mood, sleep, } \\
\text { social, physical exercise, activity, and } \\
\text { behaviour; automated sensor data. } \\
\text { - Monitoring period: } 10 \text { weeks } \\
\text { - Incentive: (Raffle) prizes }\end{array}$ & $\begin{array}{l}\text { Psychometric properties: } \\
\text { - Concurrent validity: significant moderate } \\
\text { relationship between averaged app-assessed } \\
\text { stress ratings and retrospective post-study } \\
\text { questionnaire scores on a measure of } \\
\text { perceived stress }(r=0.41, p<0.01) \\
\text { Usability: } \\
\text { - Participation rate: response rates for } \\
\text { participants who used own phones: } 65 \% \text {; } \\
\text { response rates for participants who used } \\
\text { study phones: } 72 \% \\
\text { Clinical impacts: not studied/reported }\end{array}$ \\
\hline
\end{tabular}

Tharison/control: none

Location: USA

- type not specified

name: StudentLife

Operation system: Android

access

Device: participants either used their own

Android phones (primary users) or were

users)

Incentive: (Raffle) prizes study phones: $72 \%$
Clinical impacts: not studied/reported

${ }^{a}$ The accessibility of mood-monitoring apps was assessed through a search of Google and three app stores (iTunes, Google Play and Microsoft store) in June 2016.

${ }^{b}$ Please refer to Table 2 for coefficient values.

${ }^{\mathrm{c}}$ These studies utilised the same data.

${ }^{\mathrm{d}}$ These studies utilised the same data.

e These studies utilised the same data.

${ }^{\mathrm{f}}$ These studies partly utilised the same data.

${ }^{\mathrm{g}}$ These studies utilised the same data. 
Table 2. Internal consistency coefficients across studies and domains

\begin{tabular}{|c|c|c|c|c|c|c|c|c|c|c|c|c|}
\hline \multirow[b]{3}{*}{ Authors } & \multicolumn{6}{|c|}{$\alpha$ Coefficients } & & & & \multirow{2}{*}{\multicolumn{3}{|c|}{$\frac{\Omega \text { Coefficients }}{\text { Impulsivity }}$}} \\
\hline & \multicolumn{3}{|c|}{ Positive affect } & \multicolumn{3}{|c|}{ Negative affect } & \multicolumn{3}{|c|}{ Perceived stress } & & & \\
\hline & $\mathrm{O}$ & WS & BS & $\mathrm{O}$ & WS & BS & $\mathrm{O}$ & WS & BS & $\mathrm{O}$ & WS & BS \\
\hline Ansell et al. (2015) & - & - & - & - & - & - & - & - & - & - & 0.78 & 0.96 \\
\hline Dunton et al. (2011) & 0.88 & - & - & 0.75 & - & - & - & - & - & - & - & - \\
\hline Dunton et al. (2014) & 0.87 & - & - & 0.74 & - & - & - & - & - & - & - & - \\
\hline Huh et al. (2014) & 0.65 & - & - & 0.78 & - & - & 0.73 & - & - & - & - & - \\
\hline
\end{tabular}

Note: O, Overall, WS, within-subject level, BS, between-subject level. Internal consistency coefficients values interpretation: ' $>0.9$ - excellent, $>0.8$ - good, $>0.7$ - acceptable, $>0.6$ - questionable, $>0.5$ - poor and $<0.5$ - unacceptable' (George \& Mallery, 2003, pp. 231).

a sample with high-functioning autism and Asperger's found that the app was not quite as successful in these domains. In both studies, the apps were less successful in capturing participants' thoughts.

\section{Usability of mood-monitoring apps}

\section{Participation rates}

Twenty-one studies examined participation rates, which ranged from $30 \%$ to $99 \%$. Average percentages were not computed in four studies. Instead, these studies described the mean number of diary entries per participant (Bossmann et al. 2013), between-group differences (Matthews et al. 2008b; Kauer et al. 2009), or evidence of ongoing compliance (Tregarthen et al. 2015). There was some indication that response rates were higher in studies with incentives. For example, Dennis et al. (2015) offered an incentive of $\$ 50$ per week, and had a participation rate of $89 \%$ (see Table 1 for comparative rates and incentive details). Participation rates also appeared to be affected by response fatigue. In Reid et al. (2009), for instance, response rates decreased from $91 \%$ on day 1 to $67 \%$ on day 7. Finally, participation rates were potentially affected by sample-specific characteristics. In a study with high-functioning autistic participants, Khor et al. (2014a) found a significant positive correlation between full-scale IQ and compliance rates $(r=0.46, p<0.01)$.

\section{Participants' perceptions}

Nine studies considered participants' perceptions of the apps. Three of these studies specifically referred to the 'acceptability' of apps. In Dennis et al. (2015), 95\% of adolescents felt that the EMA app 'was not too long'. Tregarthen et al. (2015) measured app utilisation data as a proxy for acceptability. There were over 100000 users over a 2-year period (with $89 \%$ using the application at least three times), which the authors interpreted as a demonstration of broad acceptability. While they did not define acceptability specifically, Reid et al. (2009) concluded that their app was 'acceptable' based on the data they captured (e.g. completion rates, participants' feedback).

Across studies, $93-100 \%$ of respondents found apps easy to learn or use (Dennis et al. 2015; Kenny et al. 2015; Sacco, 2015). In addition, participants rated apps as useful (Kenny et al. 2015), convenient, userfriendly (Bachmann et al. 2015), youth-friendly and non-invasive (Reid et al. 2009). Despite these positive experiences, technological difficulties (e.g. software crashes, reduced battery life) were reported to negatively affect user experience and participation (Loventoft et al. 2012; Huh et al. 2014; Dennis et al. 2015; Sacco, 2015). Although most young people reported a preference for mobile phone mood charting in comparison to paper diaries (Matthews et al. 2008b), not all young people preferred mobile technology (Reid et al. 2009; Scotti, 2015). Scotti (2015), e.g. found that several participants from a sub-diagnostic eating disorder sample favoured paper-and-pencil to track their data.

\section{Positive and negative clinical impacts of mood-monitoring apps}

Mental health and awareness

Five (two were from the same RCT) studies examined potential clinical impacts of the apps. Reid et al. (2011) found a significant improvement in emotional selfawareness, but no significant improvements in depression, anxiety or stress scores in youth with mental health or emotional problems. In a secondary analysis of the same RCT, Kauer et al. (2012) reported an indirect association between app use and depression 
symptoms via increased emotional self-awareness. The app, however, did not significantly reduce rumination.

Qualitative feedback from two studies also suggested that mood-monitoring apps can help improve self-awareness (Kenny et al. 2015), and self-reflection on emotions or behaviours (Sacco, 2015).

Though they did not test this premise directly, Ansell et al. (2015) hypothesised that app-based monitoring could have promoted self-awareness in participants subsequently reducing (perceived) interpersonal hostility.

In Khor et al. (2014b), parents rated their children with high-functioning autism as showing fewer symptoms of behaviour and emotional problems following use of the self-monitoring app.

\section{Treatment implications}

Five studies reported results that could have implications for the prevention and treatment of mental health problems. Mobile app data gathered by Dennis et al. (2015) were used to identify high-risk groups for substance use, which could potentially help with relapse prevention. Crooke et al. (2013) suggested that moodmonitoring apps could help investigate adolescents' motivations for drinking, thus informing the development of interventions.

Qualitative feedback from therapists suggests that the use of mobile apps could help facilitate engagement with participants suffering from various mental health problems (Matthews \& Doherty, 2011). Reid et al. (2012) reported that the Mobiletype app facilitated the assessment and management of youth mental health problems and reduced consultation time with paediatricians; the data captured enabled more individually focused consultations, which assisted in rapport building and communication.

In the third of a series of papers detailing their RCT, Reid et al. (2013) explored the potential treatment benefits of 'Mobiletype'. In comparison to the control programme, the app significantly increased general practitioners' (GPs) understanding of their patients' health and current functioning, and aided diagnoses, communication, medication and referrals. However, there was no significant effect on doctor's confidence, doctor-patient rapport or pathways to care.

Finally, in a conference paper by Loventoft et al. (2012), clinicians highlighted the usefulness of selfmonitoring when combined with therapy.

\section{Quality assessment}

Please see online Supplementary Fig. S1 for an overall depiction of the risk of bias domains across studies.

Risk of selection bias was difficult to assess in many studies, as they often lacked treatment, control or comparison groups. Three studies (all using the same RCT data) were deemed at low risk of selection bias due to a clear description of the randomisation and concealment allocation process (Reid et al. 2011, 2013; Kauer et al. 2012). Two studies were at unclear risk of selection bias because randomised sequence generation and method of allocation concealment were not sufficiently described (Matthews et al. 2008b; Reid et al. 2009). One study was considered at high risk of selection bias (Scotti, 2015) as there was no random allocation process for the control condition.

Only the RCT study (three publications) addressed the blinding of participants and personnel, and was thus considered at low risk of performance bias (Reid et al. 2011, 2013; Kauer et al. 2012). The risk of detection bias in these studies was unclear due to a lack of clarity on blinding of outcome assessments.

The risk of attrition bias was difficult to ascertain in three studies. In one study (Kenny et al. 2015), a number of participants were not included in the final sample due to restrictions on school access (no other information was available). Bossmann et al. (2013) excluded 15 participants from the final sample due to 'missing data', but did not provide further information, including whether any analyses were performed to address missing data. Reid et al. (2012) was considered at unclear risk of attrition bias, as there was no information on the participants (21\%) lost to follow-up. The remaining studies appeared to be at low risk of attrition bias. There was insufficient information to assess the risk of reporting bias in all studies but those of the RCT, which addressed pre-specified outcomes and appeared to be at low risk (Reid et al. 2011, 2013; Kauer et al. 2012). All studies appeared to be at unclear or high risk of other types of bias.

\section{Discussion}

The aim of this review was to summarise and evaluate evidence for the use of mobile mood-monitoring apps in young people (aged 10-24 years) from clinical and non-clinical populations. We specifically focused on psychometric properties, usability and clinical impacts.

\section{Psychometric properties of mood-monitoring apps}

Few studies assessed psychometric properties. There was limited evidence for reliability, with four studies demonstrating questionable to excellent levels of internal consistency. Studies examining concurrent $(n=3)$ and face $(n=2)$ validity were also sparse, making it difficult to draw firm conclusions. Face validity findings, e.g. could have been moderated by sample characteristics, e.g. reduced insight in participants with autism (Khor et al. 2014a). 
The limited assessment of psychometric properties observed in the youth literature mirrors the adult literature. Evidence for concurrent validity in adult populations is inconclusive (Depp et al. 2012; Palmier-Claus et al. 2012; Faurholt-Jepsen et al. 2014). Inconsistent methodology across these studies, e.g. momentary (Depp et al. 2012) v. retrospective assessments (Faurholt-Jepsen et al. 2014), varying periods between the event and participants' recollection of the event (Palmier-Claus et al. 2012), likely contribute to variable findings. Previous evidence suggests that real-time mood measurement methods (e.g. EMA) only have a modest correlation with retrospective assessments, such as questionnaires (Ebner-Priemer \& Trull, 2009). This leads to the conceptual question of whether retrospective measures are the most appropriate comparators when assessing the validity of mood-monitoring apps. Questionnaires measure an individual's retrospective view of their mood state over a number of days. While they are subject to recall bias, this bias incorporates other emotional processing (e.g. contexts) that the more instantaneous assessment of mood (e.g. EMA) may not capture, or at least as richly. Thus, the two assessment methods may be measuring different types of affective experience. As it is difficult to draw robust conclusions about the validity of apps using retrospective assessments, future studies should further examine psychometric properties using other sources of comparative data, e.g. active smartphone app data (i.e. app assessments) with passive sensor smartphone data (Nicholas et al. 2015; Sandstrom et al. 2016b), associations with clinical rating scales (Faurholt-Jepsen et al. 2016).

\section{Usability of mood-monitoring apps}

The usability of mood-monitoring apps was more extensively studied, and overall studies suggest that apps are usable for young people. However, there were some within- and between-study differences in participants' perceptions of apps, and participation rates.

Generally, participation rates were lower in studies where participants had mental health difficulties (Reid et al. 2011; Kauer et al. 2012), problematic drinking patterns (Kauer et al. 2009) or autism spectrum disorders - especially those with lower IQ (Khor et al. 2014a). In particular, participation levels were low for those living without set routines (Kauer et al. 2009). This is an important consideration, as youths with mood-related problems, e.g. borderline personality disorder, often have disorganised daily routines (Fleischer et al. 2012). This suggests a need to tailor apps for different clinical populations (Kauer et al. 2009).
Some studies indicated that incentives could positively influence participation rates (e.g. Ansell et al. 2015; Dennis et al. 2015). It may not be financially feasible to offer incentives in non-research settings. However, results tentatively suggest that participation rates may be better for mobile apps than traditional paper-based assessments irrespective of incentives (Matthews et al. 2008b). Participation rates for paperbased diaries are as low as $11 \%$ (Stone et al. 2003) compared with $30-99 \%$ for mood-monitoring apps in the current review. This supports that apps could lead to better adherence rates than non-digital assessment tools in young populations. Factors that could improve participation rates include the use of less intensive assessments (e.g. once-daily rather than multiple times), shorter assessments and the incorporation of staff monitoring or automatic reminders (Huh et al. 2014).

Studies from the adult literature are somewhat congruent in supporting the usability of mood-monitoring apps (Bardram et al. 2013), though evidence suggests that increasing age (e.g. 'middle age') may lower likelihood of mood-monitoring app use (Depp et al. 2012). Both adult (Palmier-Claus et al. 2013) and adolescent (Bradford \& Rickwood, 2014) populations expressed some reservations about using apps due to the perceived risk of reduced personal contact (Palmier-Claus et al. 2013).

Overall our review demonstrated that young people positively perceive apps (Reid et al. 2009) and would be willing to use this technology in real-life settings (Kenny et al. 2015; Tregarthen et al. 2015). Very few studies considered clinician perspectives on moodmonitoring apps. Matthews \& Doherty (2011) found that therapists' confidence with technology was the biggest barrier to the use of mood apps. More qualitative studies are now needed to further explore young peoples' (and clinicians') perceptions (Hollis et al. 2016) to broaden our understanding of factors pertinent to the uptake of mood-monitoring apps in real-life settings.

\section{Positive and negative clinical impacts of mood-monitoring apps}

Few of the included studies assessed the clinical impacts of the mood-monitoring apps. Although evidence was generally positive (e.g. facilitating assessment, management and GPs' understanding), most studies relied on subjective participant feedback (Sacco, 2015) rather than RCT methodology with objective outcome measures.

The preliminary evidence (Kauer et al. 2012) very tentatively suggests that electronic mood-monitoring apps could function as an intervention tool (Seko 
et al. 2014; Olff, 2015; Faurholt-Jepsen et al. 2016). Intriguingly, results from the one RCT indicated that mood-monitoring apps might reduce depression in youths by increasing their levels of emotional awareness (Kauer et al. 2012). Similarly, though in a nonexperimental study, Khor et al. (2014b) reported that self-monitoring improved parent-reported behavioural and emotional problems in participants with autism. While these results are promising, they require replication and future studies may further explore the mechanisms via which apps could potentially impact on clinical outcomes. One possibility is that mood apps could have a positive impact on clinical symptoms due to patient/participant expectations regarding their benefits. This phenomenon, coined the digital placebo effect, is an overlooked area, which also merits future investigation (Torous \& Firth, 2016).

We were unable to fully examine the potential negative impacts of mood-monitoring apps in youth populations, as they were not directly investigated in studies. However, Reid et al. (2009) found that participants did not always respond to questions truthfully to avoid having to answer further questions. Thus, this type of assessment could potentially lead to the inaccurate assessment (and treatment) of mental health problems.

A small number of adult studies report on the negative effects of mood-monitoring apps. There is some suggestion that apps may increase negative reactivity (Ainsworth et al. 2013), increase focus on negative symptoms and thoughts (Palmier-Claus et al. 2013), and potentially maintain depressive symptoms (Faurholt-Jepsen et al. 2015). Given the evidence from the adult literature, research on the possible harmful effects of app use in youths is needed before these tools are routinely used in clinical practice. Part of this endeavour should seek to identify the optimal balance between a monitoring schedule, which accurately captures affective dynamic processes, while minimising respondent workload (Bolger et al. 2003; Trull et al. 2015). This is particularly important, not only because it affects participation rates, but also because the responsibility of self-monitoring could impose a burden on young people (Shiffman et al. 2008), might result in unnecessary pressure (Lupton, 2013; Seko et al. 2014) and exacerbate mental health problems (Conner \& Reid, 2012; Faurholt-Jepsen et al. 2015).

Future work may investigate potential ethical issues surrounding the use of mood-monitoring apps. For example, their use could lead to an over-reliance on technology in young populations, which could exacerbate mental health problems (Thomée et al. 2011). There could also be information security-related risks (e.g. digital theft) that could compromise confidentiality (Prentice \& Dobson, 2014). Finally, youths could use apps as a replacement for treatment and health monitoring (Tregarthen et al. 2015). Considering the importance of the therapeutic alliance for successful treatment outcomes (Karver et al. 2006), the efficacy of smartphone apps could be reduced if they are used without clinicians' involvement (Prentice \& Dobson, 2014).

\section{Strengths and limitations}

As far as we are aware, this is the first review to systematically examine and quality assess the evidence for the psychometric properties, usability and clinical outcomes of mood-monitoring apps in youth. However, our results should be considered through the lens of a number of limitations.

First, despite undertaking a comprehensive search, there were very few high-quality studies available for inclusion in the review. There was only one primary RCT highlighting the need for more trials on the efficacy of mood-monitoring apps in young people. Indeed, our quality assessment indicated that the majority of studies included some form of bias. For example, many studies were at high or unclear risk of sampling (e.g. self-selected samples) and attrition bias. This could have affected the generalisability of our findings or led to an overestimation of positive effects, e.g. our findings may only apply to individuals with less severe psychopathology who are more likely to engage with services.

Second, studies demonstrated a great variability in terminology (especially for implementation outcomes, e.g. acceptability) making interpretations and crossstudy comparisons difficult (inconsistent terminology is also a common feature of the adult app literature). For example, we found that 'acceptability' was defined very differently across studies, ranging from proxy markers, i.e. utilisation data (Tregarthen et al. 2015) to participants' experience of burden (Dennis et al. 2015). This highlights the need for more careful delineation and measurement of implementation outcomes in future work (Proctor et al. 2011).

Third, there were large variations in samples and methodologies, again making cross-study comparisons difficult and quantitative synthesis (i.e. meta-analysis) impossible. Thus, some of our conclusions remain tentative pending further rigorous, higher quality research (e.g. RCTs).

Fourth, it should be noted that studies in this review often used apps that were specifically developed for the study, and therefore not publically available through app platforms (e.g. iTunes). Thus, there is a need for more research to assess the evidence for apps that are freely downloaded and used by youth, 
and whether their use can be incorporated into clinical care (Nicholas et al. 2015).

\section{Clinical and research implications}

Mood-monitoring apps could potentially have positive effects in both clinical and sub-clinical youth populations. Indeed, mood-monitoring apps may help youth identify and address burgeoning mental health and substance use problems (Dennis et al. 2015), and possibly utilise more adaptive coping strategies (Kauer et al. 2012). Further research is needed to examine the effects of these apps in samples with serious mental disorders, such as bipolar disorder (Grunerbl et al. 2015), borderline personality disorder (Lederer et al. 2014) and psychosis (Ben-Zeev et al. 2014; PalmierClaus et al. 2014).

Evidence, though limited, suggests that moodmonitoring apps could potentially aid diagnosis and treatment decision-making (Reid et al. 2013). Future studies should explore whether this technology could aid in the assessment of disorders that can be difficult to differentiate [e.g. borderline personality disorder, bipolar disorder (Yen et al. 2015)] by providing rich data about the timing and extent of mood fluctuations.

As technological innovations have been endorsed at a government level, integrating mood-monitoring apps within mental health services may improve access and relieve some of the strain these services are currently experiencing [e.g. by improving access to mental health treatment (Department of Health, 2013)]. However, to date, the potential positive and negative impacts of apps have not been sufficiently investigated in youth.

\section{Supplementary material}

The supplementary material for this article can be found at https://doi.org/10.1017/S0033291717001659.

\section{Acknowledgements}

MD is funded by an Economic and Social Research Council (ESRC) Collaborative Award Studentship ES/J500203/1. Funding for open access publication was kindly provided by Research Councils UK (RCUK).

\section{References}

Ainsworth J, Palmier-Claus JE, Machin M, Barrowclough C, Dunn G, Rogers A, Buchan I, Barkus E, Kapur S, Wykes T, Hopkins RS, Lewis S (2013). A comparison of two delivery modalities of a mobile phone-based assessment for serious mental illness: native smartphone application vs text-messaging only implementations. Journal of Medical Internet Research 15, e60.

Ansell EB, Laws HB, Roche MJ, Sinha R (2015). Effects of marijuana use on impulsivity and hostility in daily life. Drug and Alcohol Dependence 148, 136-142.

Bachmann A, Klebsattel C, Budde M, Riedel T, Beigl M, Reichert M, Santangelo P, Ebner-Priemer U (2015). How to use smartphones for less obtrusive ambulatory mood assessment and mood recognition. In Adjunct Proceedings of the 2015 ACM International Joint Conference on Pervasive and Ubiquitous Computing and Proceedings of the 2015 ACM International Symposium on Wearable Computers, pp. 693-702. ACM: Osaka.

Bakker D, Kazantzis N, Rickwood D, Rickard N (2016). Mental health smartphone apps: review and evidence-based recommendations for future developments. Journal of Medical Internet Research Mental Health 3, e7.

Bardram JE, Frost M, Szántó K, Faurholt-Jepsen M, Vinberg M, Kessing LV (2013). Designing mobile health technology for bipolar disorder: a field trial of the monarca system. In Proceedings of the SIGCHI Conference on Human Factors in Computing Systems, pp. 2627-2636. ACM: Paris.

Ben-Zeev D, Brenner CJ, Begale M, Duffecy J, Mohr DC, Mueser KT (2014). Feasibility, acceptability, and preliminary efficacy of a smartphone intervention for schizophrenia. Schizophrenia Bulletin 40, 1244-1253.

Ben-Zeev D, Scherer EA, Wang R, Xie H, Campbell AT (2015). Next-generation psychiatric assessment: using smartphone sensors to monitor behavior and mental health. Psychiatric Rehabilitation Journal 38, 218-226.

Bolger N, Davis A, Rafaeli E (2003). Diary methods: capturing life as it is lived. Annual Review of Psychology 54, 579-616.

Bossmann T, Kanning M, Koudela-Hamila S, Hey S, Ebner-Priemer U (2013). The association between short periods of everyday life activities and affective states: a replication study using ambulatory assessment. Frontiers in Psychology 4, 102.

Bradford S, Rickwood D (2014). Adolescent's preferred modes of delivery for mental health services. Child and Adolescent Mental Health 19, 39-45.

Buysse DJ, Reynolds CF, Monk TH, Berman SR, Kupfer DJ (1989). The Pittsburgh Sleep Quality Index: a new instrument for psychiatric practice and research. Psychiatry Research 28, 193-213.

Carver CS, Scheier MF, Weintraub JK (1989). Assessing coping strategies: a theoretically based approach. Journal of Personality and Social Psychology 56, 267-283.

Conner TS, Reid KA (2012). Effects of intensive mobile happiness reporting in daily life. Social Psychological and Personality Science 3, 315-323.

Connor-Smith JK, Compas BE, Wadsworth ME, Thomsen AH, Saltzman H (2000). Responses to stress in adolescence: measurement of coping and involuntary stress responses. Journal of Consulting and Clinical Psychology 68, 976-992.

Cristobal-Narvaez P, Sheinbaum T, Ballespi S, Mitjavila M, Myin-Germeys I, Kwapil TR, Barrantes-Vidal N (2016). Impact of adverse childhood experiences on psychotic-like symptoms and stress reactivity in daily life in nonclinical young adults. PLoS ONE 11, e0153557. 
Crooke AH, Reid SC, Kauer SD, McKenzie DP, Hearps SJ, Khor AS, Forbes AB (2013). Temporal mood changes associated with different levels of adolescent drinking: using mobile phones and experience sampling methods to explore motivations for adolescent alcohol use. Drug and Alcohol Review 32, 262-268.

Crowell SE, Beauchaine TP, Linehan MM (2009). A biosocial developmental model of borderline personality: elaborating and extending Linehan's theory. Psychological Bulletin 135, 495-510.

Dennis ML, Scott CK, Funk RR, Nicholson L (2015). A pilot study to examine the feasibility and potential effectiveness of using smartphones to provide recovery support for adolescents. Substance Abuse 36, 486-492.

Department of Health (2013). Annual Report of the Chief Medical Officer 2013, Public Mental Health Priorities: Investing in the Evidence. (https://www.gov.uk/ government/uploads/system/uploads/attachment_data/ file/413196/CMO_web_doc.pdf).

Depp CA, Ceglowski J, Wang VC, Yaghouti F, Mausbach BT, Thompson WK, Granholm EL (2015). Augmenting psychoeducation with a mobile intervention for bipolar disorder: a randomized controlled trial. Journal of Affective Disorders 174, 23-30.

Depp CA, Kim DH, de Dios LV, Wang V, Ceglowski J (2012). A pilot study of mood ratings captured by mobile phone versus paper-and-pencil mood charts in bipolar disorder. Journal of Dual Diagnosis 8, 326-332.

Donker T, Petrie K, Proudfoot J, Clarke J, Birch MR, Christensen H (2013). Smartphones for smarter delivery of mental health programs: a systematic review. Journal of Medical Internet Research 15, e247.

Dunton GF, Huh J, Leventhal AM, Riggs N, Hedeker D, Spruijt-Metz D, Pentz MA (2014). Momentary assessment of affect, physical feeling states, and physical activity in children. Health Psychology 33, 255-263.

Dunton GF, Liao Y, Intille S, Wolch J, Pentz MA (2011). Physical and social contextual influences on children's leisure-time physical activity: an ecological momentary assessment study. Journal of Physical Activity and Health $\mathbf{8}$ (Suppl. 1), S103-S108.

Ebner-Priemer UW, Trull TJ (2009). Ecological momentary assessment of mood disorders and mood dysregulation. Psychological Assessment 21, 463-475.

Faurholt-Jepsen M, Frost M, Ritz C, Christensen EM, Jacoby AS, Mikkelsen RL, Knorr U, Bardram JE, Vinberg M, Kessing LV (2015). Daily electronic self-monitoring in bipolar disorder using smartphones - the MONARCA I trial: a randomized, placebo-controlled, single-blind, parallel group trial. Psychological Medicine 45, 2691-2704.

Faurholt-Jepsen M, Frost M, Vinberg M, Christensen EM, Bardram JE, Kessing LV (2014). Smartphone data as objective measures of bipolar disorder symptoms. Psychiatry Research 217, 124-127.

Faurholt-Jepsen M, Munkholm K, Frost M, Bardram JE, Kessing LV (2016). Electronic self-monitoring of mood using IT platforms in adult patients with bipolar disorder: a systematic review of the validity and evidence. BioMed Central Psychiatry 16, 1.
Firth J, Torous J, Yung AR (2016). Ecological momentary assessment and beyond: the rising interest in e-mental health research. Journal of Psychiatric Research 80, 3-4.

Fleischer M, Schäfer M, Coogan A, Häßler F, Thome J (2012). Sleep disturbances and circadian CLOCK genes in borderline personality disorder. Journal of Neural Transmission 119, 1105-1110.

George D, Mallery P (2003). SPSS for Windows Step by Step: A Simple Guide and Reference, 11.0 Update. Allyn \& Bacon: Boston.

Grunerbl A, Muaremi A, Osmani V, Bahle G, Ohler S, Troster G, Mayora O, Haring C, Lukowicz P (2015). Smartphone-based recognition of states and state changes in bipolar disorder patients. Institute of Electrical and Electronics Engineers Journal of Biomedical and Health Informatics 19, 140-148.

Higgins JPT, Altman DG, Gøtzsche PC, Jüni P, Moher D, Oxman AD, Savović J, Schulz KF, Weeks L, Sterne JAC, Cochrane Bias Methods Group, Cochrane Statistical Methods Group (2011). The Cochrane Collaboration's tool for assessing risk of bias in randomised trials. BMJ 343, d5928.

HM Government (2011). No Health without Mental Health: a Cross-Government Mental Health Outcomes Strategy for People of All Ages. (https://www.rcpsych.ac.uk/PDF/No\% 20Health\%20without\%20Mental\%20Health.pdf).

Hollis C, Falconer CJ, Martin JL, Whittington C, Stockton S, Glazebrook C, Davies EB (2016). Annual research review: digital health interventions for children and young people with mental health problems - a systematic and meta-review. Journal of Child Psychology and Psychiatry 58, 474-503.

Huh J, Shin H, Leventhal AM, Spruijt-Metz D, Abramova Z, Cerrada C, Hedeker D, Dunton G (2014). Momentary negative moods and being with friends precede cigarette use among Korean American emerging adults. Nicotine and Tobacco Research 16, 1248-1254.

ISO/IEC 9126-1 (2001). Software Engineering - Product Quality Part 1: Quality Model. International Organization for Standardization: Geneva.

Karver MS, Handelsman JB, Fields S, Bickman L (2006). Meta-analysis of therapeutic relationship variables in youth and family therapy: the evidence for different relationship variables in the child and adolescent treatment outcome literature. Clinical Psychology Review 26, 50-65.

Kauer SD, Reid SC, Crooke AHD, Khor A, Hearps SJC, Jorm AF, Sanci L, Patton G (2012). Self-monitoring using mobile phones in the early stages of adolescent depression: randomized controlled trial. Journal of Medical Internet Research 14, e67.

Kauer SD, Reid SC, Sanci L, Patton GC (2009). Investigating the utility of mobile phones for collecting data about adolescent alcohol use and related mood, stress and coping behaviours: lessons and recommendations. Drug and Alcohol Review 28, 25-30.

Kenny R, Dooley B, Fitzgerald A (2015). Feasibility of 'CopeSmart': a telemental health app for adolescents. Journal of Medical Internet Research Mental Health 2, e22.

Khor AS, Gray KM, Reid SC, Melvin GA (2014a). Feasibility and validity of ecological momentary assessment in 
adolescents with high-functioning autism and Asperger's disorder. Journal of Adolescence 37, 37-46.

Khor AS, Melvin GA, Reid SC, Gray KM (2014b). Coping, daily hassles and behavior and emotional problems in adolescents with high-functioning autism/Asperger's disorder. Journal of Autism and Developmental Disorders 44, 593-608.

Lederer N, Grechenig T, Baranyi R (2014). unCUT: bridging the gap from paper diary cards towards mobile electronic monitoring solutions in borderline and self-injury. In 2014 IEEE 3rd International Conference on Serious Games and Applications for Health (SeGAH), pp. 1-8. IEEE: Rio de Janeiro.

Loventoft PK, Norregaard LB, Frokjaer E (2012). Designing daybuilder: an experimental app to support people with depression. In Proceedings of the 12th Participatory Design Conference: Exploratory Papers, Workshop Descriptions, Industry Cases - vol. 2, pp. 1-4. ACM: Roskilde.

Lupton D (2013). Quantifying the body: monitoring and measuring health in the age of mHealth technologies. Critical Public Health 23, 393-403.

Marwaha S, Balbuena L, Winsper C, Bowen R (2015). Mood instability as a precursor to depressive illness: a prospective and mediational analysis. Australian and New Zealand Journal of Psychiatry 49, 557-565.

Matthews M, Doherty G (2011). In the mood: engaging teenagers in psychotherapy using mobile phones. In Proceedings of the SIGCHI Conference on Human Factors in Computing Systems, pp. 2947-2956. ACM: Vancouver.

Matthews M, Doherty G, Coyle D, Sharry J (2008a). Designing mobile applications to support mental health interventions. In Handbook of Research on User Interface Design and Evaluation for Mobile Technology (ed. J. Lumsden), pp. 635-656. IGI Global: Hershey.

Matthews M, Doherty G, Sharry J, Fitzpatrick C (2008b). Mobile phone mood charting for adolescents. British Journal of Guidance and Counselling 36, 113-129.

Moher D, Liberati A, Tetzlaff J, Altman DG, The PRISMA Group (2009). Preferred reporting items for systematic reviews and meta-analyses: the PRISMA statement. Annals of Internal Medicine 151, 264-269, w264.

Myin-Germeys I, Klippel A, Steinhart H, Reininghaus U (2016). Ecological momentary interventions in psychiatry. Current Opinion in Psychiatry 29, 258-263.

Naslund JA, Marsch LA, McHugo GJ, Bartels SJ (2015). Emerging mHealth and eHealth interventions for serious mental illness: a review of the literature. Journal of Mental Health 24, 321-332.

NCCMH (2014). E-therapies Systematic Review for Children and Young People with Mental Health Problems. NCCMH: London.

Nicholas J, Larsen ME, Proudfoot J, Christensen H (2015). Mobile apps for bipolar disorder: a systematic review of features and content quality. Journal of Medical Internet Research 17, e198.

Ofcom (2015). The Communications Market Report. (https:// www.ofcom.org.uk/_data/assets/pdf_file/0022/20668/ cmr_uk_2015.pdf).

Olff M (2015). Mobile mental health: a challenging research agenda. European Journal of Psychotraumatology 6, 27882.
Palmier-Claus JE, Ainsworth J, Machin M, Barrowclough C, Dunn G, Barkus E, Rogers A, Wykes T, Kapur S, Buchan I, Salter E, Lewis SW (2012). The feasibility and validity of ambulatory self-report of psychotic symptoms using a smartphone software application. BioMed Central Psychiatry 12, 172.

Palmier-Claus JE, Rogers A, Ainsworth J, Machin M, Barrowclough C, Laverty L, Barkus E, Kapur S, Wykes T, Lewis SW (2013). Integrating mobile-phone based assessment for psychosis into people's everyday lives and clinical care: a qualitative study. BioMed Central Psychiatry 13, 34 .

Palmier-Claus JE, Taylor PJ, Ainsworth J, Machin M, Dunn G, Lewis SW (2014). The temporal association between self-injurious thoughts and psychotic symptoms: a mobile phone assessment study. Suicide and Life-Threatening Behavior 44, 101-110.

Paris J (2004). Borderline or bipolar? Distinguishing borderline personality disorder from bipolar spectrum disorders. Harvard Review of Psychiatry 12, 140-145.

Prentice JL, Dobson KS (2014). A review of the risks and benefits associated with mobile phone applications for psychological interventions. Canadian Psychology/Psychologie Canadienne 55, 282-290.

Proctor E, Silmere H, Raghavan R, Hovmand P, Aarons G, Bunger A, Griffey R, Hensley M (2011). Outcomes for implementation research: conceptual distinctions, measurement challenges, and research agenda. Administration and Policy in Mental Health 38, 65-76.

Reid SC, Kauer SD, Dudgeon P, Sanci LA, Shrier LA, Patton GC (2009). A mobile phone program to track young people's experiences of mood, stress and coping: development and testing of the mobiletype program. Social Psychiatry and Psychiatric Epidemiology 44, 501-507.

Reid SC, Kauer SD, Hearps SJC, Crooke AHD, Khor AS, Sanci LA, Patton GC (2011). A mobile phone application for the assessment and management of youth mental health problems in primary care: a randomised controlled trial. BioMed Central Family Practice 12, 131.

Reid SC, Kauer SD, Hearps SJC, Crooke AHD, Khor AS, Sanci LA, Patton GC (2013). A mobile phone application for the assessment and management of youth mental health problems in primary care: health service outcomes from a randomised controlled trial of mobiletype. BioMed Central Family Practice 14, 84.

Reid SC, Kauer SD, Khor AS, Hearps SJC, Sanci LA, Kennedy AD, Patton GC (2012). Using a mobile phone application in youth mental health: an evaluation study. Australian Family Physician 41, 711-714.

Sacco GR (2015). Developing a Smartphone Application for Depression: Tracking Risk and Wellness Factors. M.A. University of Delaware.

Sandstrom GM, Lathia N, Mascolo C, Rentfrow PJ (2016a). Opportunities for smartphones in clinical care: the future of mobile mood monitoring. Journal of Clinical Psychiatry 77, e135-e137.

Sandstrom GM, Lathia N, Mascolo C, Rentfrow PJ (2016b). Putting mood in context: Using smartphones to examine 
how people feel in different locations. Journal of Research in Personality (http://dx.doi.org/10.1016/j.jp.2016.06.004).

Schwartz JE, Neale J, Marco C, Shiffman SS, Stone AA (1999). Does trait coping exist? A momentary assessment approach to the evaluation of traits. Journal of Personality and Social Psychology 77, 360-369.

Scotti JF (2015). School-based DBT skills groups for adolescent eating disorders and body image concerns: a pilot study. Ph.D. University of Montana.

Seko Y, Kidd S, Wiljer D, McKenzie K (2014). Youth mental health interventions via mobile phones: a scoping review. Cyberpsychology, Behavior and Social Networking 17, 591-602.

Shiffman S, Stone AA, Hufford MR (2008). Ecological momentary assessment. Annual Review of Clinical Psychology 4, 1-32.

Stone AA, Shiffman S, Schwartz JE, Broderick JE, Hufford MR (2003). Patient compliance with paper and electronic diaries. Controlled Clinical Trials 24, 182-199.

Thomée S, Härenstam A, Hagberg M (2011). Mobile phone use and stress, sleep disturbances, and symptoms of depression among young adults - a prospective cohort study. BioMed Central Public Health 11, 66.

Torous J, Firth J (2016). The digital placebo effect: mobile mental health meets clinical psychiatry. Lancet Psychiatry 3, 100-102.

Torous J, Powell AC (2015). Current research and trends in the use of smartphone applications for mood disorders. Internet Interventions 2, 169-173.

Tregarthen JP, Lock J, Darcy AM (2015). Development of a smartphone application for eating disorder self-monitoring. International Journal of Eating Disorders 48, 972-982.

Trull TJ, Lane SP, Koval P, Ebner-Priemer UW (2015). Affective dynamics in psychopathology. Emotion Review 7, 355-361. van Knippenberg RJM, de Vugt ME, Ponds RW, Myin-Germeys I, van Twillert B, Verhey FRJ (2016). Dealing with daily challenges in dementia (deal-id study): an experience sampling study to assess caregiver functioning in the flow of daily life. International Journal of Geriatric Psychiatry (https://dx.doi.org/10.1002/gps.4552).

Walsh S, Golden E, Priebe S (2016). Systematic review of patients' participation in and experiences of technology-based monitoring of mental health symptoms in the community. BMJ Open 6, e008362.

Wang R, Chen F, Chen Z, Li T, Harari G, Tignor S, Zhou X, Ben-Zeev D, Campbell AT (2014). StudentLife: assessing mental health, academic performance and behavioral trends of college students using smartphones. In Proceedings of the 2014 ACM International Joint Conference on Pervasive and Ubiquitous Computing, pp. 3-14. ACM: Seattle.

Watson D, Clark LA, Tellegen A (1988). Development and validation of brief measures of positive and negative affect: the PANAS scales. Journal of Personality and Social Psychology 54, 1063-1070.

Wenze SJ, Miller IW (2010). Use of ecological momentary assessment in mood disorders research. Clinical Psychology Review 30, 794-804.

World Health Organisation (1986). Young people's health: a challenge for society - Report of a WHO Study Group on Young People and Health for All by the Year 2000. World Health Organisation Technical Report Series 731. World Health Organisation: Geneva.

Yen S, Frazier E, Hower H, Weinstock LM, Topor DR, Hunt J, Goldstein TR, Goldstein BI, Gill MK, Ryan ND, Strober M, Birmaher B, Keller MB (2015). Borderline personality disorder in transition age youth with bipolar disorder. Acta Psychiatrica Scandinavica 132, 270-280. 